\title{
Liquid Biopsy in Advanced Gastric Malignancy and Molecular Targeted Therapy; a Case Report and Update Pertaining Anti HER2-Neu Therapy
}

\author{
AL-Kadhimi M, Al-Dawoodi T, Peguero J ${ }^{*}$ and Campos LT
}

Oncology Consultants, Research Department Houston, Texas, USA

${ }^{*}$ Corresponding author: Pegureo J, M.D., Oncology Consultants, International Cancer center, 2130 West Holcombe Blvd. 10 ${ }^{\text {th }}$ Floor, Houston, Texas, USA 77030, Fax: 713.600.0070, Tel: 713.600.0900, E-mail: jpeguero@oncologyconsultants.com

Citation: AL-Kadhimi M, Al-Dawoodi T, Peguero J, Campos LT (2016) Liquid Biopsy in Advanced Gastric Malignancy and Molecular Targeted Therapy; a Case Report and Update Pertaining Anti HER2-Neu Therapy. J Cancer Sci Clin Oncol 3(2): 202. doi: 10.15744/2394-6520.3.202

Received Date: August 05, 2016 Accepted Date: September 07, 2016 Published Date: September 09, 2016

\begin{abstract}
Human epidermal growth factor receptor 2 (HER2) is responsible for the pathogenesis and poor outcomes of several types of cancers, including advanced gastric and gastroesophageal junction cancer. Molecular-targeted drugs on the other hand, such as trastuzumab, prolong overall survival and progression-free survival in HER2-positive gastric cancer. The purpose of the case report is to evaluate the impact of delivering trastuzumab in advanced gastric cancer with concomitant HER2 mutation and amplification.

Keywords: Gastric cancer; Human epidermal growth factor receptor 2; CT scan; Trastuzumab; Biopsy

List of Abbreviations: HER2: Human Epidermal Growth Factor Receptor 2; GC: Gastric Cancer; OS: Overall Survival; PIK3CA: Phosphatidylinositol -4, 5- Bisphosphate 3-Kinase, Catalytic subunit Alpha; EGFR: Epidermal Growth Factor Receptor; CT: Computerized tomography; PET: Positron emission test; ER: Emergency room; TP53: Tumor protein p53; CTNB1: $\beta$-catenin; CDK6: Cell Division Protein Kinase 6; SUV: Standardized uptake values
\end{abstract}

\section{Introduction}

Gastric cancer (GC) is the fourth most common type of cancer and the second leading cause of cancer-related deaths in the world [1]. Most patients suffering from the advanced, inoperable or metastatic stage of the disease have 5- year survival rates and are approximately 30\% (of the cancer population?) [2]. Validated chemotherapeutic regimens such as fluoropyrimidine and/or platinum-based therapies failed to improve the prognosis of advanced GC that remains poor, with a median overall survival (OS) being around 1 year $[3,4]$.

Fragments of DNA shed by cancers into the bloodstream can potentially be used as a non-invasively screening method for earlystage cancers, monitor responses to treatment and explains why some cancers are resistant to therapies. For most neoplasms, a tissue biopsy is quite challenging in that it is costly, painful, unreachable, insufficient amount of staining or potentially risky for the patient. All these are good reasons to learn about cancer through blood and to get excited about the possibility of carrying out liquid biopsies. The development of non-invasive methods to detect and monitor cancers continues to be a major challenge in oncology. Cell-free circulating tumor DNA (ctDNA) and circulating tumor cells (CTCs) are plasma sources of tumor DNA that have been investigated for non-invasive detection and monitoring of patient tumors but have not been analyzed or directly compared across multiple tumor types. Although the current Food and Drug Administration (FDA)-approved liquid biopsy measures intact CTCs to give a prognosis of overall survival, the potential predictive value of ctDNA is much more exciting. ctDNA liquid biopsy allows us to understand specifically what kind of molecular changes are happening in the tumor in real time, which is a very big step beyond where CTCs are today in clinical terms.

Therefore, there is an urgent need for targeted-driven approaches toward deregulated molecular signaling pathways in advanced GC such as phosphatidylinositol -4, 5- bisphosphate 3-kinase, catalytic subunit alpha (PIK3CA) pathway or epidermal growth factor receptor (EGFR) pathway. HER2 is the first validated treatment target in HER2-positive GC. HER2 amplification is reported in $7-34 \%$ of tumor cases [5,6]. Although anti-HER2 therapy such as Trastuzumab confers clinical benefit on GC patients, its efficacy was shown to be unsatisfactory due to primary or acquired resistance [7-9]. The ToGA trial [7] reported a prolongation of median OS by a modest 2.7 months (from 11.1 months to 13.8 months) with Trastuzumab. 


\section{Case Report}

A 72-year-old Filipino male initially had an 8 months history of right upper quadrant pain. This prompted an ER visit where he underwent a CT scan of the abdomen and pelvis. Imaging revealed a large retroperitoneal mass involving the posterior aspect of the pancreatic head and uncinate process. A CA 19-9 level was normal at that time. A CT guided biopsy of this retroperitoneal mass was obtained, and the preliminary pathology report showed adenocarcinoma. The tissue specimen was insufficient for immunohistochemical staining. Due to diagnostic uncertainty, the patient on June $5^{\text {th }} 2015$, underwent a PET CT scan that revealed a $10.5 \mathrm{~cm}$ by $11.0 \mathrm{~cm}$ retroperitoneum mass with SUVs peaking at 13.9. It also showed a $3.4 \mathrm{~cm}$ by $2.8 \mathrm{~cm}$ mass in the posterior aspect of the stomach with SUVs peaking at 4.9. Hypermetabolic retroperitoneal, and bilateral iliac chain lymph nodes. Osseous metastasis was present at T11 with SUVs peaking at 8.5.

Due to clinical difficulty in performing a repeat biopsy, we performed liquid biopsy, which revealed ERBB2 mutation (D769Y) and amplification, CTNNB1 (S33F) mutation, TP53 (M246V) mutation, MYC amplification and CDK6 amplification. After introducing four cycles of Docetaxel, 5-Fluorouracil and Oxaliplatin, and Trastuzumab, PET scan demonstrated progression of his disease and based on this, we switched therapy to Cisplatin, Irinotecan, and trastuzumab. The patient delivered 2 cycles and could not tolerate the regimen, in spite of the fact that PET scan demonstrated excellent response. So we discontinued Cisplatin and continued Irinotecan and Trastuzumab. CT scan of chest, abdomen, and pelvis obtained after four cycles of biweekly Irinotecan and Trastuzumab showed an excellent response, and complete remission.

\section{Discussion}

We present a case of advanced metastatic gastric adenocarcinoma, which was treated with Trastuzumab combined with chemotherapy in the first and second line settings. We know from ToGA trial that molecular targeted therapy is directed at HER2 (ERBB2) amplification and overexpression by immunohistochemical staining and fluorescence in vitro hybridization. In this case, we used next generation sequencing of circulating DNA from a liquid biopsy and found it to have ERBB2 mutation and amplification. We treated with Trastuzumab as a molecular targeted therapy and obtained full response and complete remission clinically and radiologically. The patient is free of disease for 6 months since the first PET scan was obtained. From this aspect, we have two interesting observations: the first one is the use of liquid biopsy technique and the other, the use of molecular targeted therapy to obtain complete response in advanced gastric malignancy. We hypothesized that advanced gastric malignancy having concurrent ERBB2 mutation (D769Y) and amplification may potentially give better response to targeted therapy with anti HER2, than having only mutation or amplification.

\section{Conclusion}

Gastric cancer (GC) is the fourth most common type of cancer and the second leading cause of cancer-related death in the world [1]. Using liquid biopsy in advanced gastric malignancy and the use of molecular targeted therapy with Trastuzumab, in future may obviate the use of immunohistochemical staining and fluorescence in vitro hybridization on solid biopsy.

\section{Acknowledgments}

We wish to thank Peguero J, M.D, Campos LT, M.D, for providing editorial support.

\section{Conflict of interest statement}

I do not have any conflict of interest to disclose. Dr. Peguero J serves in the speaker bureau for Foundation Medicine, Bristol Myers Squibb and Bayer. He directs phase one and two programs that funded by pharma-industry at Oncology Consultants Research Department.

\section{References}

1. Jemal A, Center MM, DeSantis C, Ward EM (2010) Global patterns of cancer incidence and mortality rates and trends. Cancer Epidemiol Biomarkers Prev 19: 1893-907.

2. Siegel R, Naishadham D, Jemal A (2013) Cancer statistics, 2013. CA Cancer J Clin 63: 11-30.

3. Koizumi W, Narahara H, Hara T, Takagane A, Akiya T, et al. (2008) S-1 plus cisplatin versus S-1 alone for first-line treatment of advanced gastric cancer (SPIRITS trial): a phase III trial. Lancet Oncol 9: 215-21.

4. Cunningham D, Starling N, Rao S, Iveson T, Nicolson M, et al. (2008) Capecitabine and oxaliplatin for advanced esophagogastric cancer. N Engl J Med 358: 36-46.

5. Gravalos C, Jimeno A (2008) HER2 in gastric cancer: a new prognostic factor and a novel therapeutic target. Ann Oncol 19: 1523-9.

6. Hofmann M, Stoss O, Shi D, Büttner R, van de Vijver M, et al. (2008) Assessment of a HER2 scoring system for gastric cancer: results from a validation study. Histopathology 52: 797-805.

7. Bang YJ, Van Cutsem E, Feyereislova A, Chung HC, Shen L, et al. (2010) Trastuzumab in combination with chemotherapy versus chemotherapy alone for treatment of HER2-positive advanced gastric or gastro-oesophageal junction cancer (ToGA): a phase 3, open-label, randomised controlled trial. Lancet 376: 68797. 
8. Satoh T, Xu R-H, Chung HC, Sun G-P, Doi T, et al. (2014) Lapatinib plus paclitaxel versus paclitaxel alone in the second- line treatment of HER2-amplified advanced gastric cancer in Asian populations: TyTAN--a randomized, phase III study. J Clin Oncol 32: 2039-49.

9. Hecht JR, Bang Y-J, Qin S, Chung H-C, Xu J-M, et al. (2013) Lapatinib in combination with capecitabine plus oxaliplatin (CapeOx) in HER2-positive advanced or metastatic gastric, esophageal, or gastroesophageal adenocarcinoma (AC): The TRIO-013/LOGiC trial. J Clin Oncol 31: LBA4001.

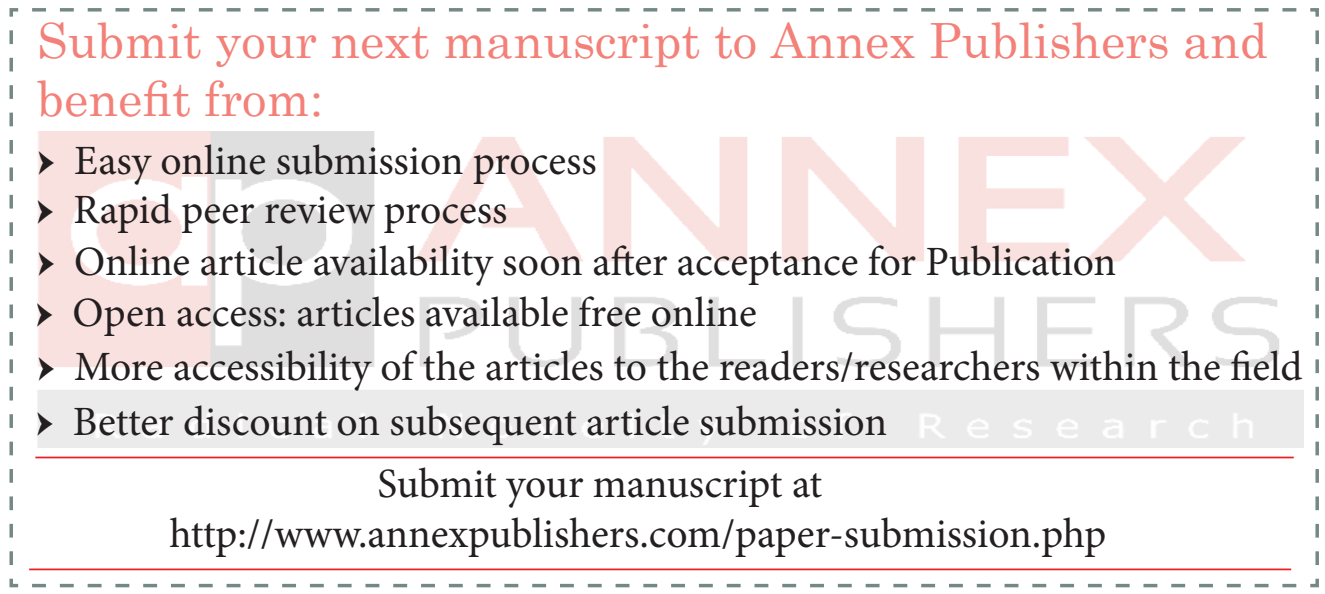

